\title{
Completeness of prostate cancer staging in the Danish Cancer Registry, 2004-2009
}

This article was published in the following Dove Press journal:

Clinical Epidemiology

16 August 2012

Number of times this article has been viewed

\author{
Mary Nguyen-Nielsen' \\ Trine Frøslev' \\ Søren Friis ${ }^{2}$ \\ Michael Borre ${ }^{3}$ \\ Niels Harving ${ }^{4}$ \\ Mette Søgaard ${ }^{1,5}$ \\ 'Department of Clinical Epidemiology, \\ Aarhus University Hospital, Aarhus, \\ ${ }^{2}$ Danish Cancer Society Research \\ Center, Danish Cancer Society, \\ Copenhagen, ${ }^{3}$ Department of \\ Urology, Aarhus University Hospital \\ at Skejby, Aarhus, ${ }^{4}$ Department of \\ Urology, Aalborg Hospital, Aarhus \\ University Hospital, Aalborg, \\ ${ }^{5}$ Department of Clinical Microbiology, \\ Aalborg Hospital, Aarhus University \\ Hospital, Aalborg, Denmark
}

Correspondence: Mary Nguyen-Nielsen Department of Clinical Epidemiology, Aarhus University Hospital, Olof Palmes Allé 43-45, 8200 Aarhus N, Denmark

Tel +4587167607

Fax +4587167215

Email mary.n.nielsen@dce.au.dk
Objective: To investigate the completeness of TNM (Tumor-Node-Metastasis) staging for prostate cancer (PC) in the Danish Cancer Registry (DCR).

Methods: We identified 20,184 men registered with first-time PC in the DCR between 2004 and 2009. These patients were linked to the Danish National Patient Register to obtain data on comorbidity according to the Charlson Comorbidity Index (CCI). We calculated the completeness and corresponding 95\% confidence intervals (CI) of TNM staging overall and by the individual components. We also defined a clinically-based algorithm classifying PC into four stage categories: localized, regional, distant, and unknown.

Results: The overall completeness of TNM staging was 34.2\% (95\% CI: 0.34-0.35). TNM completeness improved gradually over time reaching $41.2 \%$ in 2009 . TNM completeness decreased substantially with age from $75.0 \%$ among patients $0-39$ years to $11.3 \%$ among patients 80 years or older. Similarly, completeness decreased with increasing comorbidity level from $37.6 \%$ among patients with low CCI to $20.3 \%$ among those with high CCI. When classifying T1 cancer as a complete registration regardless of missing $\mathrm{N}$ or $\mathrm{M}$ stage, the overall TNM completeness increased to $48.7 \%$ (95\% CI: 0.48-0.49). According to the clinically-based staging algorithm, $70.5 \%$ of $\mathrm{PC}$ cases could be categorized into a definite clinical stage.

Conclusion: One-third of PC patients had a complete registration of all TNM components in the DCR. Although TNM completeness improved over time, older age and high comorbidity were consistently associated with missing TNM staging. Research and monitoring based on cancer registries such as the DCR should account for missing TNM staging. Failing to do so could otherwise lead to biased results of stage-specific analyses.

Keywords: prostate cancer, neoplasm staging, TNM

\section{Introduction}

Prostate cancer (PC) is the most common nonskin cancer among men in Western countries. ${ }^{1,2}$ However, there is great variation in prognosis across countries and according to clinical stage. ${ }^{3}$ The overall five-year relative survival for PC in Denmark was $62 \%-68 \%$ during $2001-2006,{ }^{4}$ whereas the corresponding figure was approximately 78\% in other Nordic countries during 1999-2003. ${ }^{5}$ In the United States, the relative five-year survival for PC was estimated to be $99 \%$ overall; $100 \%$ for localized PC and 29\% for metastatic PC during 2001-2007. ${ }^{1}$ With the dramatic differences in PC survival across countries and according to PC stage, knowledge of clinical staging has become increasingly important in both survival analyses and treatment strategies. ${ }^{6,7}$

Cancer staging is based on the TNM (Tumor- Node-Metastasis) classification system as outlined by the International Union against Cancer and the American Joint 
Committee on Cancer. ${ }^{8,9}$ Exact T staging of PC (ie, T1-4) is particularly important because cancer grading (ie, Gleason scoring) and pre-operative prostate-specific antigen (PSA) is incorporated in the assessment of localized PC into low, intermediate, or high risk tumors. ${ }^{10,11}$ Data on TNM classification, but not including Gleason scores and PSA values, have been registered in the Danish Cancer Registry (DCR) since 2004. The overall validity and completeness of cancer registration in the DCR is high, ${ }^{12,13}$ but the completeness of TNM cancer staging is unknown. Missing information in cancer registries may lead to biased results in epidemiological studies and monitoring, especially if the missing data are not distributed randomly. Thus, to ascertain the magnitude and direction of the potential bias, it is important to understand and examine the characteristics of unstaged cancer cases and how these differ from the staged cases. ${ }^{14}$ The present study investigated the completeness of TNM staging for PC, stratified by age, comorbidity, and year of diagnosis in the DCR.

\section{Material and methods}

We performed this study in Denmark (population 5.5 million inhabitants). All residents in Denmark are provided with free, tax-supported medical care. Since 1968, the Danish Civil Registration System has assigned a unique 10-digit civil registration number to all Danish residents, which encodes date-of-birth and gender. ${ }^{15}$ This number is used in all Danish registries, allowing for unambiguous individual-level data linkage.

\section{Ascertainment of patients with PC}

From the DCR, we identified all patients who had a primary cancer diagnosis of PC [International Classification of Disease, 10th revision (ICD-10) code C61] recorded in the DCR between 2004 and 2009. The DCR has recorded information on incident cancers in the Danish population since 1943. ${ }^{12,16}$ Cancer diagnoses have been registered according to the ICD for Oncology (ICD-O) during the period 1978-2003 and the ICD-10 since 2004, but converted into ICD-10 codes for the entire period encompassing 1978-2009. ${ }^{16}$ From the DCR, we obtained information on date of diagnosis, age, gender, and TNM codes. We categorized cancer stage as localized, regional, distant or unknown based on a clinical algorithm using all T, N, and M components (Appendix 1). Missing data were allowed in the three clinical stage categories if the available information provided sufficient and clinically meaningful information to categorize cases. The "unknown" stage category consisted primarily of locally advanced tumors (T3 or T4) with unknown $\mathrm{N}$ and/or $\mathrm{M}$ stage.

\section{Comorbidity}

Data on comorbidities were obtained from the Danish National Patient Register (DNPR). ${ }^{17}$ The DNPR contains data on all admissions to nonpsychiatric hospitals in Denmark since 1977 and all outpatient contacts since 1995, including the civil registration number, date of admission/contact, and diagnosis codes. We identified pre-existing comorbidity using the Charlson Comorbidity Index (CCI). ${ }^{18,19}$ The CCI was based on all Charlson comorbidities (excluding PC) registered within 10 years preceding the date of PC diagnosis. CCI levels were categorized as 0 (low), 1-2 (medium), and $3+$ (high).

\section{Statistical analysis}

We calculated the proportion of complete TNM registrations and corresponding 95\% confidence intervals (CI), overall and by each component individually (ie, T, N, and M). Registration of TNM was defined as complete if the patient had received the following codes: T0-4, N0-3, and M0-1. A registration of $\mathrm{Tx}, \mathrm{Nx}$, or $\mathrm{Mx}$ was defined as an unknown stage (ie, information on tumor size, lymph node metastases, and distant metastases were not available or could not be assessed). We stratified completeness by gender, age (0-39 years, 40-59 years, 60-79 years, and $\geq 80$ years), year of cancer diagnosis, and CCI level $(0,1-2,3+)$. We defined T1-Nx-Mx as a complete TNM registration and repeated the analyses. In addition, we repeated the analyses restricting it to histologically verified PC diagnoses. Finally, we categorized PC patients into localized, regional, distant, or unknown stages using the above mentioned clinical algorithm (Appendix 1). Analyses were performed using Statistical Analysis Software (v 9.2; SAS Institute, Inc, Cary, NC, USA).

\section{Results}

We identified 20,184 men with primary PC between 2004 and 2009. The median age at diagnosis was 71 years (interquartile range, 65 to 77 years). The overall completeness of TNM registration was $34.2 \%$ (95\% CI: $0.34-0.35)$, whereas $65.8 \%$ (95\% CI: 0.65-0.66) had a registration of Tx, Nx, and/or Mx (Table 1). Completeness was lowest for " $\mathrm{N}$ " at $36.7 \%$, compared with $88.8 \%$ for $\mathrm{T}$, and $60.0 \%$ for M. Notably, the completeness of the TNM registration improved gradually over the six-year study period from $28.4 \%$ (95\% CI: $0.27-0.30$ ) in 2004 to $41.2 \%$ (95\% CI: $0.40-0.43$ ) in 2009. However, the completeness of TNM registration decreased with advancing age from 75.0\% (95\% CI: 0.28-0.97) among patients aged $0-39$ years to $11.3 \%$ (95\% CI: $0.10-0.12$ ) 
Table I Completeness of the TNM registration among prostate cancer patients in the Danish Cancer Registry (2004-2009)

\begin{tabular}{|c|c|c|c|c|c|c|c|c|}
\hline & \multicolumn{2}{|c|}{ TNM completeness } & \multicolumn{2}{|c|}{ T completeness } & \multicolumn{2}{|c|}{$\mathbf{N}$ completeness } & \multicolumn{2}{|c|}{ M completeness } \\
\hline & $\%(n)$ & $95 \% \mathrm{Cl}$ & $\%(n)$ & $95 \% \mathrm{Cl}$ & $\%(n)$ & $95 \% \mathrm{Cl}$ & $\%(n)$ & $95 \% \mathrm{Cl}$ \\
\hline Total & 34.2 (6899) & $0.34-0.35$ & $88.8(17917)$ & $0.88-0.89$ & $36.7(7407)$ & $0.36-0.37$ & $60.0(12119)$ & $0.59-0.61$ \\
\hline \multicolumn{9}{|c|}{ Year of diagnosis } \\
\hline 2004 & 28.4 (77I) & $0.27-0.30$ & $85.3(23 \mid 3)$ & $0.84-0.87$ & $30.9(837)$ & $0.29-0.33$ & $58.6(1590)$ & $0.57-0.60$ \\
\hline 2005 & $29.0(877)$ & $0.27-0.31$ & $89.3(2704)$ & $0.88-0.90$ & $31.4(952)$ & $0.30-0.33$ & $58.7(1776)$ & $0.57-0.60$ \\
\hline 2006 & 32.0 (989) & $0.30-0.34$ & $88.6(2739)$ & $0.87-0.90$ & $34.5(1067)$ & $0.33-0.36$ & $59.1(1827)$ & $0.57-0.61$ \\
\hline 2007 & $33.8(|26|)$ & $0.32-0.35$ & $90.0(3361)$ & $0.89-0.91$ & $36.0(1344)$ & $0.34-0.38$ & $59.9(2238)$ & $0.58-0.61$ \\
\hline 2008 & $37.5(1391)$ & $0.36-0.39$ & $89.9(3335)$ & $0.89-0.91$ & $40.2(1489)$ & $0.39-0.42$ & $60.4(224 I)$ & $0.59-0.62$ \\
\hline 2009 & $41.2(1610)$ & $0.40-0.43$ & $88.7(3465)$ & $0.88-0.90$ & $44.0(1718)$ & $0.42-0.46$ & $62.6(2447)$ & $0.61-0.64$ \\
\hline \multicolumn{9}{|l|}{ Age group } \\
\hline $0-39$ years & $75.0(3)$ & $0.28-0.97$ & $75.0(3)$ & $0.28-0.97$ & $75.0(3)$ & $0.28-0.97$ & $100.0(4)$ & $0.56-1.00$ \\
\hline $40-59$ years & $50.2(1039)$ & $0.48-0.52$ & $93.6(1938)$ & $0.92-0.95$ & $53.0(1097)$ & $0.5 \mathrm{I}-0.55$ & $72.7(1505)$ & $0.7 I-0.75$ \\
\hline 60-79 years & $37.3(5466)$ & $0.37-0.38$ & 90.5 (13267) & $0.90-0.91$ & $39.9(5847)$ & $0.39-0.41$ & $62.5(9164)$ & $0.62-0.63$ \\
\hline$\geq 80$ years & II.3 (391) & $0.10-0.12$ & 78.5 (2709) & $0.77-0.80$ & I3.3 (460) & $0.12-0.14$ & $41.9(1446)$ & $0.40-0.44$ \\
\hline \multicolumn{9}{|l|}{ CCl score } \\
\hline Low (0) & $37.6(4973)$ & $0.37-0.38$ & 90.5 (I 1976) & $0.90-0.91$ & $40.1(5305)$ & $0.39-0.41$ & $62.5(8266)$ & $0.62-0.63$ \\
\hline Medium (I-2) & $29.7(1632)$ & $0.28-0.31$ & $87.6(4820)$ & $0.87-0.88$ & $32.0(1764)$ & $0.31-0.33$ & $56.3(3096)$ & $0.55-0.58$ \\
\hline High $(\geq 3)$ & $20.3(294)$ & $0.18-0.22$ & $77.4(1121)$ & $0.75-0.80$ & $23.3(338)$ & $0.21-0.26$ & $52.3(757)$ & $0.50-0.55$ \\
\hline
\end{tabular}

Abbreviations: $\mathrm{Cl}$, confidence interval; $\mathrm{CCl}$, Charlson Comorbidity Index; $\mathrm{M}$, metastasis; $\mathrm{n}$, number; $\mathrm{N}$, node; $\mathrm{T}$, tumor; TNM, tumor-node-metastasis.

among those aged 80 years or older. Likewise, completeness decreased with increasing CCI level from $37.6 \%(95 \% \mathrm{CI}$ : $0.37-0.38$ ) among patients with a low CCI level to $20.3 \%$ (95\% CI: $0.18-0.22)$ among those with a high CCI level.

When T1-Nx-Mx was included as a complete TNM registration, the overall completeness improved to $48.7 \%$ (95\% CI: 0.48-0.49). By contrast, restricting the analysis to patients with histologically verified PC $(80.7 \%)$ only marginally improved the overall TNM completeness to $36.5 \%$ (95\% CI: 0.36-0.37).When applying our staging algorithm, we found that localized PC accounted for $52.6 \%$, regional PC for $2.5 \%$, and distant PC for $15.4 \%$ of the cases (Table 2), yielding an overall clinical staging completeness of $70.5 \%$. Patients aged 80 years or older and patients with a high CCI level had the highest proportion of unknown staging, $47.1 \%$ and $36.0 \%$, respectively.

\section{Discussion}

Consistent with previous studies,${ }^{20-22}$ completeness of TNM registration decreased substantially with increasing age and high comorbidity. We found that only $34.2 \%$ of PC patients had a complete registration of all three $\mathrm{T}, \mathrm{N}$, and $\mathrm{M}$ components in the DCR and this low proportion was mainly due to incomplete registration of $\mathrm{N}$-status. A recent study by Merrill et al examined the proportion of unstaged cancer for 18 cancer sites in the United States based on data from the Surveillance, Epidemiology, and End Results Program of the National Cancer Institute. ${ }^{20}$ The authors found that the level of unstaged cancer was higher among the elderly, nonmarried, and for cancer types associated with poor survival (eg, hepatic, esophageal, and pancreatic cancer). Likewise, Koroukian et al examined staging for breast cancer, colorectal cancer, and PC, and showed that the level of unstaged cancer increased with increasing age and those with higher comorbidity were 4 to 5 times more likely to have incomplete cancer staging than those with few comorbidities. ${ }^{23}$

Table 2 Staging of prostate cancer patients in the Danish Cancer Registry by year of prostate cancer diagnosis, sex, age group, and comorbidity level

\begin{tabular}{|c|c|c|c|c|}
\hline & \multicolumn{4}{|c|}{ Cancer stage } \\
\hline & $\begin{array}{l}\text { Localized } \\
\text { n (\%) }\end{array}$ & $\begin{array}{l}\text { Regional } \\
\text { n (\%) }\end{array}$ & $\begin{array}{l}\text { Distant } \\
\mathrm{n}(\%)\end{array}$ & $\begin{array}{l}\text { Unknown } \\
\text { n (\%) }\end{array}$ \\
\hline Total $(n=20,184)$ & $10616(52.6)$ & $504(2.5)$ & $3118(15.4)$ & $5946(29.5)$ \\
\hline \multicolumn{5}{|l|}{ Year of diagnosis } \\
\hline 2004 & I 258 (46.4) & $54(2.0)$ & $569(21.0)$ & $831(30.6)$ \\
\hline 2005 & I 474 (48.7) & 7I (2.3) & $585(19.3)$ & $898(29.7)$ \\
\hline 2006 & I565 (50.6) & $80(2.6)$ & $507(16.4)$ & $94 \mathrm{I}(30.4)$ \\
\hline 2007 & $2027(54.3)$ & $85(2.3)$ & $549(14.7)$ & $1074(28.8)$ \\
\hline 2008 & $2024(54.6)$ & $98(2.6)$ & 497 (I3.4) & $1089(29.4)$ \\
\hline 2009 & $2268(58.0)$ & $116(3.0)$ & $4 I I(10.5)$ & $1113(28.5)$ \\
\hline \multicolumn{5}{|c|}{ Age group (years) } \\
\hline $0-39$ & $3(75.0)$ & $0(0.0)$ & I (25.0) & $0(0.0)$ \\
\hline $40-59$ & $1418(68.5)$ & $87(4.2)$ & $209(10.1)$ & $357(17.2)$ \\
\hline $60-79$ & $8240(56.2)$ & $407(2.8)$ & $2046(14.0)$ & $3965(27.1)$ \\
\hline$\geq 80$ & $955(27.7)$ & $10(0.3)$ & $862(25.0)$ & $1624(47.1)$ \\
\hline \multicolumn{5}{|l|}{ CCI score } \\
\hline Low (0) & $7375(55.7)$ & $383(2.9)$ & I832 (13.8) & $3642(27.5)$ \\
\hline Medium (I-2) & $2665(48.4)$ & $108(2.0)$ & 949 (17.2) & $1782(32.4)$ \\
\hline High $(\geq 3)$ & $576(39.8)$ & $13(0.9)$ & $337(23.3)$ & $522(36.0)$ \\
\hline
\end{tabular}

Abbreviations: $\mathrm{CCl}$, Charlson Comorbidity Index; n, number. 
Klassen et al investigated staging among 22,217 men with PC in the Maryland Cancer Registry. ${ }^{21}$ These authors found that older age, black race, and higher median income increased the likelihood of missing cancer staging. Another study of "all cancer types," based on 1,907 nursing home residents in Michigan, USA, suggested that comorbidity was not associated with a higher proportion of unstaged cancer; however, the majority of the patients in this study were over 86 years old, thus reducing the generalizability of these findings due to selection bias. ${ }^{24}$ Finally, Radespiel-Troger et al investigated the completeness of PC staging in the Bavarian Cancer Registry and reported an overall registration completeness of $96 \%$, and the proportions of missing $\mathrm{T}, \mathrm{N}$, and $\mathrm{M}$ were $15 \%, 37 \%$, and $49 \%$, respectively. ${ }^{25}$

The strengths of our study are: (1) its population-based design; (2) the large sample size of over 20,000 PC patients; (3) the comprehensiveness of the Danish medical registries together with the ability to link data across multiple registries; and (4) the nature of the uniform health care system in Denmark that ensures equal access to free health care to all residents. Furthermore, the registration of cancer incidence is virtually complete in the DCR, ${ }^{12,13,16}$ and the positive predictive value of coding in the DNPR for comorbid diseases (CCI) was recently shown to be high. ${ }^{19}$

The main limitation of our study is that it only investigated the level of TNM completeness; we did not assess the actual validity of registration (eg, by medical record review). It would be relevant to proceed with validation studies to investigate the quality and validity of TNM staging in the DCR. It is also important to note that a given TNM stage in the DCR reflects the stage at the time of initial diagnosis/registration. Also, our stage categorization (into localized, regional, or distant PC) was based on a simplified algorithm which only took into consideration $\mathrm{T}, \mathrm{N}$, and $\mathrm{M}$ stage, and thus did not include Gleason scores or PSA values as otherwise outlined by the International Union against Cancer and the American Joint Committee on Cancer 2010 guidelines for PC stage grouping. ${ }^{8-10}$ A new nationwide clinical database, DaProCaData, has recently been established in Denmark and is projected to provide detailed data on Gleason scores, cancer staging, and lifestyle variables. ${ }^{26}$ Moreover, an alternative clinical staging of PC into localized, locally advanced, and distant PC might be more appropriate than our TNM-based staging into localized, regional, and distant PC since the distinction between localized and locally advanced PC correlates better with current treatment measures. For localized PC, it would have been ideal to perform stratification according to the classification defined by the D'Amico Risk Group into low, intermediate, and high level of risk. ${ }^{27}$ However, this would require additional information on pre-diagnostic PSA levels and Gleason scores, which are not available in the DCR. A recent study investigating data completeness in the Surveillance, Epidemiology, and End Results database of the National Cancer Institute and PC staging based on the D'Amico staging showed that $33 \%$ had incomplete registration of D'Amico variables (PSA, Gleason, and/or T stage) ${ }^{28}$ Similar to our findings, elderly PC patients were also found to have the highest level of missing data when using the DARG classification. ${ }^{28}$

The reasons for the low completeness of TNM staging for PC in the DCR are most likely multifactorial and may be related to age, comorbidity, patient/doctor preferences, and current clinical guidelines. It has been suggested that with increasing age and comorbidity, patients increasingly refrain from further diagnostic measures. ${ }^{20,22}$ The limited registration of "N" likely results from the current clinical guidelines for $\mathrm{PC}$ and the diagnostic process related hereto. Staging of "N" for PC requires either surgical procedures or advanced imaging of the lymph nodes, which are not performed in all patients. The European Association of Urology guidelines on PC only recommend N-staging when "potentially curative treatment is planned for." ${ }^{29}$ Thus, if we had designed our staging algorithm in accordance with this, we would have found a lower proportion of patients with an unknown cancer stage since missing $\mathrm{N}$-stage accounted for the majority of the missing T-N-M components. Furthermore, prostate biopsies are associated with a relative high risk of bleeding and infection and CT-imaging has recently been shown to have low sensitivity and specificity in detecting dissemination to the pelvic lymph nodes. ${ }^{30}$ Likewise, extended pelvic lymph node dissection and its therapeutic benefit in the staging and management of $\mathrm{PC}$ is much debated. ${ }^{31}$ By contrast, standard diagnostic procedures for breast cancer include "sentinel node" dissection, resulting in a higher proportion of $\mathrm{N}$ registrations for breast cancer patients simply due to standard clinical practice.

When we defined T1-Nx-Mx as a complete TNM registration, the overall TNM completeness improved to $48.7 \%$. This proportion more accurately reflects TNM completeness in the DCR. In the case of a T1 PC tumor with no clinical signs of metastatic spread to the lymph nodes or to distant sites, the clinician will typically not proceed with further diagnostic testing. For such a T1 tumor, it is inferred that there is no spread outside the solid tumor, but $\mathrm{Nx}$ and $\mathrm{Mx}$ 
must be registered since $\mathrm{N} 0$ and $\mathrm{M} 0$ status has not technically been confirmed. Consequently, the registration of T1-Nx-Mx typically represents a complete TNM staging based on clinical evaluation. Likewise, when using the algorithm to classify PC cases into localized, regional, or distant stage, $70.5 \%$ of all PC patients could be staged in the DCR. These results are clinically meaningful and perhaps better reflect the true completeness of cancer staging in the DCR.

Exclusion of nonhistologically verified PC had little impact on the overall results. Compared to other common cancers registered in the DCR (ie, breast, lung and colon cancer), PC had the highest proportion of nonhistologically verified cancer diagnosis (19.3\%). This is expectable, given current clinical practice, the typical slow progression of the majority of PC cases, and the potential risks associated with invasive diagnostic procedures. The diagnosis of PC relies on a combination of digital rectal examination, PSA, and eventually histology or imaging (eg, MRI or bone scintigraphy). The benefit of histological verification and complete TNM cancer staging may simply not outweigh the potential harms.

In conclusion, one-third of PC patients had complete TNM staging in the DCR. When applying our clinical staging algorithm, $70.5 \%$ of all PC cases could be staged as localized, regional or distant metastatic. However, TNM completeness was consistently poorer with older age and high comorbidity. Our findings from the DCR highlight the potential for selection bias when data is missing. Missing or unknown data should therefore be accounted for (eg, by multiple imputation methods) when conducting stage-specific analyses in the DCR or other cancer registries.

\section{Acknowledgments}

This project was funded by the Regional Clinical Epidemiological Monitoring Initiative for Central and North Denmark Regions. A sincere thanks to Christina Gade Jespersen, MD, for her input on the clinical staging algorithm.

\section{Disclosure}

The authors declare no conflicts of interest.

\section{References}

1. Siegel R, Naishadham D, Jemal A. Cancer statistics, 2012. CA Cancer J Clin. 2012;62(1):10-29.

2. Danish Cancer Registry. Copenhagen: National Board of Health. updated 2010. Available from: http://www.sst.dk/Udgivelser/2011/ Cancerregisteret2010.aspx. Accessed January 1, 2012.

3. Coleman MP, Quaresma M, Berrino F, et al. Cancer survival in five continents: a worldwide population-based study (CONCORD). Lancet Oncol. 2008;9(8):730-756.
4. Storm HH, Gislum M, Kejs AM, Engholm G. Survival of Danish cancer patients 1995-2006. Ugeskr Laeger. 2010;172(33):2213-2217.

5. Bray F, Klint A, Gislum M, et al. Trends in survival of patients diagnosed with male genital cancers in the Nordic countries 1964-2003 followed up until the end of 2006. Acta Oncol. 2010; 49(5):644-654.

6. Newschaffer CJ, Otani K, McDonald MK, Penberthy LT. Causes of death in elderly prostate cancer patients and in a comparison nonprostate cancer cohort. J Natl Cancer Inst. 2000;92(8):613-621.

7. Ketchandji M, Kuo YF, Shahinian VB, Goodwin JS. Cause of death in older men after the diagnosis of prostate cancer. J Am Geriatr Soc. 2009;57(1):24-30

8. Page DL, Fritz AG, Balch CM, et al. AJCC Cancer Staging Manual, 6th ed. New York: Springer; 2002.

9. Sobin LH, Gospodarowicz MK, Wittekind C. TNM Classification of Malignant Tumours, 7th ed. Chichester, West Sussex, UK: WileyBlackwell; 2010

10. Cheng L, Montironi R, Bostwick DG, Lopez-Beltran A, Berney DM. Staging of prostate cancer. Histopathology. 2012;60(1):87-117.

11. Cowherd SM. Tumor staging and grading: a primer. Methods Mol Biol. 2012;823:1-18.

12. Storm HH, Michelsen EV, Clemmensen IH, Pihl J. The Danish Cancer Registry - history, content, quality and use. Dan Med Bull. 1997;44(5): 535-539.

13. Jensen AR, Overgaard J, Storm HH. Validity of breast cancer in the Danish Cancer Registry. A study based on clinical records from one county in Denmark. Eur J Cancer Prev. 2002;11(4):359-364.

14. Vach W, Blettner M. Biased estimation of the odds ratio in case-control studies due to the use of ad hoc methods of correcting for missing values for confounding variables. Am J Epidemiol. 1991;134(8): 895-907.

15. Pedersen CB. The Danish Civil Registration System. Scand J Public Health. 2011;39(Suppl 7):22-25.

16. Gjerstorff ML. The Danish Cancer Registry. Scand J Public Health. 2011;39(Suppl 7):42-45.

17. Lynge E, Sandegaard JL, Rebolj M. The Danish National Patient Register. Scand J Public Health. 2011;39(Suppl 7):30-33.

18. Charlson ME, Pompei P, Ales KL, MacKenzie CR. A new method of classifying prognostic comorbidity in longitudinal studies: development and validation. J Chronic Dis. 1987;40(5):373-383.

19. Thygesen SK, Christiansen CF, Christensen S, Lash TL, Sørensen HT. The predictive value of ICD-10 diagnostic coding used to assess Charlson comorbidity index conditions in the population-based Danish National Registry of Patients. BMC Med Res Methodol. 2011; 11:83.

20. Merrill RM, Sloan A, Anderson AE, Ryker K. Unstaged cancer in the United States: a population-based study. BMC Cancer. 2011; 11:402.

21. Klassen AC, Curriero F, Kulldorff M, Alberg AJ, Platz EA, Neloms ST. Missing stage and grade in Maryland prostate cancer surveillance data, 1992-1997. Am J Prev Med. 2006;30(Suppl 2):S77-S87.

22. Worthington JL, Koroukian SM, Cooper GS. Examining the characteristics of unstaged colon and rectal cancer cases. Cancer Detect Prev. 2008;32(3):251-258.

23. Koroukian SM, Xu F, Beaird H, Diaz M, Murray P, Rose JH. Complexity of care needs and unstaged cancer in elders: a populationbased study. Cancer Detect Prev. 2007;31(3):199-206.

24. Bradley CJ, Clement JP, Lin C. Absence of cancer diagnosis and treatment in elderly Medicaid-insured nursing home residents. $J$ Natl Cancer Inst. 2008;100(1):21-31.

25. Radespiel-Troger M, Geiss K, Gartig-Daugs A, Meyer M. Registration completeness and tumour-node-metastases category distribution in prostatic and testicular cancer in Bavaria. Eur J Cancer Prev. 2008; 17(3):230-237.

26. Danish Urological Cancer Group. Skejby, Denmark: Aarhus University Hospital; updated 2012. Available from: http://www.ducg.dk/default. asp?page=136. Accessed March 3, 2012. 
27. D'Amico AV, Whittington R, Malkowicz SB, et al. Biochemical outcome after radical prostatectomy, external beam radiation therapy, or interstitial radiation therapy for clinically localized prostate cancer. JAMA. 1998;280(11):969-974.

28. Elliott SP, Johnson DP, Jarosek SL, Konety BR, Adejoro OO, Virnig BA. Bias due to missing SEER data in D'Amico risk stratification of prostate cancer. J Urol. 2012;187(6):2026-2031.

29. Heidenreich A, Aus G, Bolla M, et al. EAU guidelines on prostate cancer. Eur Urol. 2008;53(1):68-80.
30. Briganti A, Abdollah F, Nini A, et al. Performance characteristics of computed tomography in detecting lymph node metastases in contemporary patients with prostate cancer treated with extended pelvic lymph node dissection. Eur Urol. 2011;61(6):1132-1138.

31. Briganti A, Blute ML, Eastham JH, et al. Pelvic lymph node dissection in prostate cancer. Eur Urol. 2009;55(6):1251-1265. 


\section{Appendix table}

Appendix I Algorithm for prostate cancer staging according to the TNM classification in the Danish Cancer Registry

\begin{tabular}{|c|c|}
\hline Stage & TNM-codes \\
\hline \multirow[t]{3}{*}{ Localized } & TI-4, x NO MO \\
\hline & TI-2 N0 Mx \\
\hline & TI-2 Nx M0, x \\
\hline Regional & $\mathrm{TI}-4, \times \mathrm{NI}, \mathrm{MO}$ \\
\hline \multirow[t]{3}{*}{ Distant } & $\mathrm{TI}-4, x \mathrm{NI}, \mathrm{MI}$ \\
\hline & $\mathrm{TI}-4, \mathrm{x}$ No MI \\
\hline & $\mathrm{TI}-4, \times \mathrm{N} \times \mathrm{MI}$ \\
\hline \multirow[t]{3}{*}{ Unknown } & $\mathrm{T} 3-4, \times \mathrm{N} \times \mathrm{M} 0, \mathrm{x}$ \\
\hline & T3-4, x N0 Mx \\
\hline & $\mathrm{TI}-4, \mathrm{x}$ NI Mx \\
\hline
\end{tabular}

Notes: According to UICC/AJCC guidelines, Tis, Ta, N2, and N3 are not used for PC. ${ }^{9-11}$ We identified I 44 PC cases who were registered with these classifications; these cases were categorized as "unknown." Likewise eight cases registered with "TO" were also categorized as "unknown."

Abbreviations: PC, prostate cancer; TNM, tumor-node-metastasis; UICC/AJCC, International Union against Cancer and the American Joint Committee on Cancer.

\section{Publish your work in this journal}

Clinical Epidemiology is an international, peer-reviewed, open access journal focusing on disease and drug epidemiology, identification of risk factors and screening procedures to develop optimal preventative initiatives and programs. Specific topics include: diagnosis, prognosis, treatment, screening, prevention, risk factor modification, systematic

Submit your manuscript here: http://www.dovepress.com/clinical-epidemiology-journa

\section{Dovepress}

reviews, risk \& safety of medical interventions, epidemiology \& biostatical methods, evaluation of guidelines, translational medicine, health policies \& economic evaluations. The manuscript management system is completely online and includes a very quick and fair peer-review system, which is all easy to use. 